Mr. Patterson assumes a contradiction where none exists and then proposes an artificial way out.

E. A. ECKHARDT

RANDAL Morgan Laboratory, Philladelphia, Pa.

\section{THE THIRD LAW OF MOTION AND "INERTIA REACTION"}

The recent article by $\mathrm{Mr}$. Andrew $\mathrm{H}$. Patterson in Science for March 16, 1917, impels me to add to the discussion of questions in mechanics something that I have tried to make clear to students. It is along the line of $\mathrm{Mr}$. Fulcher's article of November 24, and concerns the confusion between the third law of motion, the second law, and D'Alembert's principle.

Mr. Patterson appears to object to teaching that "to every action there is always an equal and contrary action" or that "forces always occur in pairs" and at the same time that an " unbalanced force" produces an acceleration. There is surely no inconsistency in this, since the "pairs" of forces or the action and reaction act on different bodies, say $A$ and $B$, then if no other bodies are acting upon them, there will be an unbalanced force on each, and each will be accelerated, but in opposite directions. Evidently another pair of forces may act between $B$ and $C$ such that on the whole the forces on $B$ exactly balance, and yet $A$ will be left with an accelerated motion. On the other hand, while it is clear from writing the equation representing the second law of motion in the form $F-M a=0$, that if a force equal to the mass times the acceleration should act on the body in the opposite direction to the impressed force, these forces would be in equilibrium, this is not a case of the third law, which specifies that the forces considered act between two bodies and not on one and the same body. If for a system one adds the idea (D'Alembert's principle?), that the internal actions and reactions of any system of bodies are in equilibrium among themselves, a special case of the third law, one obtains the more general statement that if forces equal to the several masses times their respective accelerations were applied, etc., a form which is useful in the handling of problems, but which does not imply that such forces are acting and does not call for the idea of "inertia reactions."

The case where "inertia reaction" is most frequently drawn in, in connection with action and reaction is the instance of an object being whirled around on the end of a string. Now when one explains the motion of the moon about the earth as due to the action of the gravitational force on the moon directed towards the earth, one looks for the "reaction" in a gravitational force on the earth directed toward the moon, but not a force on the moon, and this reaction on the earth has nothing to do with the mass $X$ acceleration of the moon, but would be the same if the moon were at rest in the position which it has at any instant. Is not the same true for the ball and string? Consider the case where a person grasps the ball by a hook at the end of a diameter, and pulls on a cord at the other end with the force $F$, the ball as well as the cord is strained, and we may say that the ball is pulling on the string and the string on the ball (the third law), in virtue of this strain. Now let go at the one end, in order to continue to apply a force $F$ the hand must be moved with the same acceleration which the ball has in order to keep the string stretched, and would not the ball in the neighborhood of the string remain strained as before and hence the forces between ball and string be of the same nature as before? Now suppose the ball swung around the head, as Mr. Patterson suggests, would not the ball still remain strained and would it not pull on the string with a force which would be exactly the same as if the ball were at rest, but in the same state of strain? If so why bring in an inertia reaction? In the illustration of the porter pushing a cart, as long as he actually pushes there is an equal counter force on him, but in the one case the push on the cart may be balanced by friction, and in the other it would be an unbalanced force on the cart. Actually if friction suddenly ceased would not the porter probably notice that the force with which he was pushing had suddenly diminished, and 
that he had to. hurry up to push at all? It would seem to me to be true in this case also that the push back on him would be the same if the cart were in the same state of strain and at rest.

If the point of view brought forward here is correct it would seem to me desirable to leave out of any elementary discussion of mechanics an "inertia reaction."

\section{Mount Holyoke CoLLege}

Elizabeth R. Laird

\section{AN ADDITIONAL NOTE ON "THE OOLITIC AND PISOLITIC BARITE FROM THE SARATOGA OIL FIELD, TEXAS"}

ABout three years ago the writer wrote a description of some barite of unusual type from the Saratoga Oil Field, Texas. ${ }^{1} \quad$ Specimens of this mineral have been brought to the surface in pumping, and they have been found, in all cases reported to the writer, at a depth around 1,200 feet, indicating that they probably have their source in a definite geological horizon. At the time the above-mentioned paper was written it was supposed that the concretions of this mineral originated with the sands in which they were found but there was no definite information on the subject.

In discussing this matter a short time ago with Mr. E. G. Woodruff, he stated that at least some of these concretions undoubtedly formed in the wells after they were equipped, because they had been found reaching a quarter of an inch in diameter, in a well with a screen on the tubing, the mesh of which was altogether too small to admit a concretion of the size stated. He kindly sent the writer an assortment of specimens of various shapes and sizes from other wells in the same field as those previously described and of approximately of the same depth. Tests with the blow-pipe and specific gravity determinations show that the composition of the concretions is almost identical to that of those previously described. A number were examined for nuclei, but in most cases no definite nucleus could be found. When a nucleus is present

1 Oölitic and Pisolitic Barite from the Saratoga Oil Field, Texas," by E. S. Moore, Bull. of the Geol. Soc. of Amer., Vol. 25, pp. 77-79, 1914. it consists of earthy material made up mostly of clay and barite and this mass is often stained with iron ioxide which gives the center of the concentration a brownish tint.

This additional information is interesting from the standpoint of its bearing on the origin of concretions. It would appear to be practically impossible for bacteria or other low types of life, which are believed to play an important part in the origin of oölites, to exist in a liquid with such strong antiseptic properties as those of warm petroleum containing considerable sulphuric acid. It would seem to demonstrate that living organisms are not essenial to the development of oollites and that these may form where precipitation is taking place in an agitated solution, in the absence of life. E. S. MOORE

The Pennsylfania State College, State Coliege, Pa.

\section{SCIENTIFIC BOOKS}

Ocean Magnetic Observations, 1905-1916, and Reports on Special Researches. By L. A. BAuER, Director, with the collaboration of W. J. Peters, J. A. Fleming, J. P. Ault and W. F. G. Swann. Washington, D. O., 1917. Carnegie Institution. Pp. vii +447 . This large and handsome volume is the third of the series issued by the department of terrestrial magnetism of the Carnegie Institution and contains full reports of all the magnetic work of the department at sea during the past eleven years. The two preceding volumes deal with the observations on land for the periods 1905-1910 and 1910-1913 respectively.

In 1905 the wooden brigantine Galilee was chartered at San Francisco and fitted up for magnetic observations with the purpose of making a preliminary survey of the Pacific Ocean which was at that time " nearly a blank as regards magnetic observations." In the course of three years, this vessel cruised 63,834 nautical miles and, magnetically speaking, put the Pacific Ocean "on the map." In addition to the great number of valuable and accurate observations which were accumulated, these cruises of the Galilee afforded an opportunity for testing and improving magnetic instru- 